\title{
The role of soy and soy isoflavones on women's fertility and related outcomes: an update
}

\author{
Gianluca Rizzo ${ }^{1}$ (1), Alessandra Feraco ${ }^{2,3}$, Maximilian Andreas Storz ${ }^{4} *$ (1) and Mauro Lombardo ${ }^{3}$ (1) \\ ${ }^{1}$ Independent Researcher, Via Venezuela 66, 98121 Messina, Italy \\ ${ }^{2}$ Laboratory of Cardiovascular Endocrinology, IRCCS San Raffaele Roma, 00166 Rome, Italy \\ ${ }^{3}$ Department of Human Sciences and Promotion of the Quality of Life, San Raffaele Roma Open University, 00166 Rome, Italy \\ ${ }^{4}$ Center for Complementary Medicine, Department of Internal Medicine II, Faculty of Medicine, University of Freiburg, Freiburg, Germany
}

(Received 30 January 2022 - Accepted 7 February 2022)

Journal of Nutritional Science (2022), vol. 11, e17, page 1 of 17

doi:10.1017/jns.2022.15

Abstract

Soy is a key food in human nutrition. It is widely used in eastern traditional cuisine and it has recently diffused among self-conscious and vegetarian diets. The success of soy mainly depends on versatility and supposed healthy properties of soy foods and soy components. Meanwhile, the possible influence on endocrine system, in particular by isoflavones, raised concerns among some researchers. The present paper aims to conduct a review of available data on the effect of soy, soy foods and soy components on women's fertility and related outcomes. Eleven interventional studies, eleven observational studies and one meta-analysis have been selected from the results of queries. A weak, not clinically relevant effect has been highlighted on cycle length and hormonal status. However, a suggestive positive influence has been shown among women with fertility issues and during assisted reproductive technologies. Overall, soy and soy components consumption do not seem to perturb healthy women's fertility and can have a favourable effect among subjects seeking pregnancy. However, because of the paucity of studies exploring the impact of soy intake on women's fertility, as well as the limited population sample size, the frequently incomplete specimens' collection to investigate all cycle phases and the insufficient characterisation of participants, the evidence is suggestive and it needs further in-depth research taking into account all these aspects.

Key words: Assisted: Fecundability: Fertility: Infertility: Isoflavones: Phytoestrogens: Reproductive techniques: Soy: Soybeans: Soy foods

\section{Introduction}

Soy is a very popular food and its consumption is part of the traditional cuisine of South-East Asian countries. However, even in the West, it is currently widely used, especially due to its versatility in plant-based products for health purposes and vegetarian diets ${ }^{(1)}$. Interest in soy is particularly driven by its possible beneficial effects on human health. Soy consumption is supposed to have protective effects against cardiovascular disease by cholesterol-lowering and blood pressure improvement action and in the prevention of cancer or diabetes and it also supports bone health and the management of menopause symptoms ${ }^{(2-8)}$.
Soy contains numerous phytochemicals that can be responsible for these positive effects through multiple mechanisms. Many of its components show an antioxidant activity that can at least partially explain its effectiveness ${ }^{(9)}$. In particular, soy contains numerous non-isoflavone constituents such as phytic acid, triterpenes and sterols, Bowman-Birk protease inhibitors, unsaturated fatty acids, saponins, inositol phosphates, proteins, peptides such as lunasin; ${ }^{(10)}$ nevertheless, soy isoflavones have attracted much attention in the last years for its estrogenic as well as non-hormonal properties ${ }^{(11)}$.

Isoflavones are non-steroidal compounds with a chemical structure similar to endogenous estrogens and for this reason, they are defined as phytoestrogens: a functional classification

* Corresponding author: Maximilian Andreas Storz, email maximilian.storz@uniklinik-freiburg.de 
that also includes lignans, coumestans and stilbenes ${ }^{(12)}$. Notably, these latter compounds are present in several foods such as legumes, cereals and seeds, whereas soy is almost the only source of isoflavones in human diet. Isoflavones show several biological properties, acting as selective tissue estrogenic activity regulators (STEARs), thanks to the differential distribution pattern of estrogen receptors in body tissues ${ }^{(13)}$ and the differentiated affinity between the two isoforms of estrogen receptors, called alpha and beta. Moreover, isoflavones act as selective estrogen receptor modulators (SERMs) showing both agonist and antagonist effects on ER, with subsequent estrogenic, anti-estrogenic or even neutral effects ${ }^{(14)}$.

Regarding isoflavones, the equol metabolite derives from the precursor daidzein by the action of intestinal bacteria. The individual conversion capacity, equol-competence, offers a useful tool for estimating the biological effect of these compounds ${ }^{(15)}$. Individuals who are not equol-producers have likely limited response to isoflavone intake ${ }^{(16)}$. Even if the exact conversion mechanism has not been characterised yet, a limited conversion capacity in Western populations (about $25 \%$ ) has been highlighted, as opposed to the greater competence of Asian populations (50\%), estimated through urinary equol excretion ${ }^{(17)}$. For this reason, in clinical studies, the nationality and ethnicity of participants may be relevant for the assessment of potential conflicting effects of soy intake.

Soy isoflavones seem to act also through a non-genomic regulation, activating specific cellular signalling pathways ${ }^{(18)}$. Finally, they show antioxidant activity: a shared property among polyphenols ${ }^{(19)}$. The beneficial efficacy of soy is often attributed to the presence of isoflavones, capable of mitigating the excesses of endogenous estrogens, through the competition with estrogen receptors or by the activation of receptors, in the presence of low levels of endogenous estrogens. On the other hand, many perplexities have been raised about possible negative mechanisms leading to endocrine disruptor effects ${ }^{(20)}$.

Consequently, it is plausible that research efforts have been aimed at evaluating the effects of soy, especially isoflavones, on human fertility and hormonal regulation. As for males, a 2010 meta-analysis highlighted the safety of soy on fertility outcomes $^{(21)}$, recently confirmed by an updated meta-analysis on this topic ${ }^{(22)}$. However, the specific effect of soy intake on women's fertility has not yet been systematically evaluated. The purpose of this review is to collect currently available data in literature, summarising the possible interaction between soy, soy foods and components of soy (in particular isoflavones) on aspects concerning women's fertility and related outcomes.

\section{Methods}

A systematic consultation of literature was launched on four search engines (PubMed, ScienceDirect, Cochrane Trials Library and ClinicalTrials.gov) using the following keywords:

('Soy' OR ‘Soy Foods' OR ‘Soybeans' OR 'Genistein' OR 'Daidzein' OR 'Isoflavones' OR 'Phytoestrogens') AND ('Fertility' OR 'Infertility' OR 'Fecundability').
Keywords were searched in titles and abstracts and combined with $\mathrm{MeSH}$ terms, where available, adapting the query format based on the search engine used. No restrictions were applied using filters and results were collected from search engines by the inception through 4 April 2021. The obtained results were evaluated for duplicates and then screened for titles and abstracts information. For the remaining papers, the full texts were retrieved for the final evaluation and inclusion in the summary.

Articles concerning reviews, case series, case studies, nonhuman studies, in vitro studies, studies on males, editorials, letters to editor, conference abstracts, book's chapters, non-English papers, studies with no-soy isoflavones and studies with outcomes not pertinent to fertility were excluded. In addition, full-text bibliographic lists from selected papers were screened to retrieve further relevant articles. The procedure was carried out following the most recent PRISMA guidelines $^{(23)}$.

\section{Results and discussion}

A total of 834 entries were obtained following search engine queries (PubMed: 381; ScienceDirect: 392; Cochrane Library Trials: 30 and ClinicalTrials.gov: 31). Following the removal of eighty-four duplicates, the selection was made through titles, abstracts and full-text reading. Finally, twelve entries were identified and ten additional articles were obtained after the consultation of full-text bibliographic lists. A total number of twenty-two experimental articles plus a meta-analysis was used for the final synthesis. The detailed selection process is highlighted in Fig. 1.

The obtained meta-analysis was included in the final summary because it assessed aspects relating to the topic of this review. This allowed to exclude the already summarised articles in meta-analysis from a detailed discussion. The results of selected manuscripts were grouped according to the outcomes used, for a clear comparison. Manuscripts exploring multiple aspects were discussed in different paragraphs, where deemed necessary.

The clinical studies selection included one retrospective study, two cross-sectional studies, eight longitudinal cohort studies, five parallel-designed interventional studies and six longitudinal interventional studies. A list of the selected clinical studies with their characteristics is summarised in Table 1.

\section{Fertility}

Fertility is defined by the number of offspring produced by an individual. Consequently, the absence of fertility, called infertility, is a disease characterised by the failure to establish a clinical pregnancy and it can depend on various factors including predisposition and behavioural/environmental aspects. The fertility concept differs from the fecundity one, which refers to reproductive potential and depends on reproductive physiology, from the production of gametes phase to the ability to carry a pregnancy to term. Therefore, the lack of fecundity is called sterility ${ }^{(47)}$. However, the terms are often interchangeably, being closely associated with the possibility of giving birth 


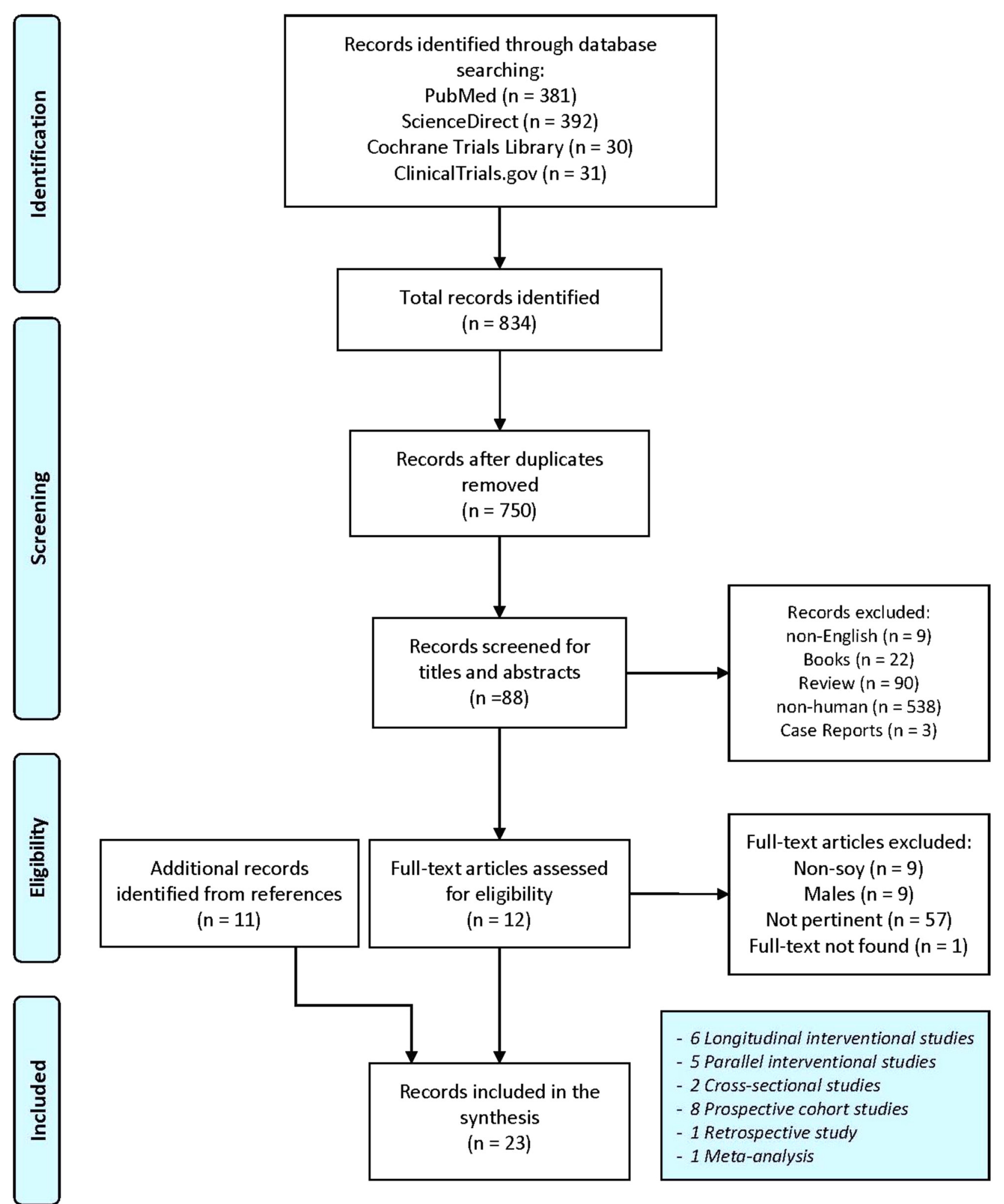

Fig. 1. Flowchart for studies selection. Adapted from Moher et al. ${ }^{(24)}$.

to children. Recently, the anti-Müllerian hormone (AMH) concentrations have proved to be a useful tool for predicting female fertility, especially because it is independent of the cycle phase ${ }^{(48)}$.

In 2005, Kohama and colleagues published a short communication about a 6 months clinical trial on thirty-six Japanese women with secondary amenorrhea (or anovulation) ${ }^{(33)}$. Six grams per day of black soybean powder were administered to the intervention group, whereas thirty-four individuals received no treatment as a control group. After the intervention period, four patients became pregnant and twelve patients showed ovulation improvements based on ultrasonography 


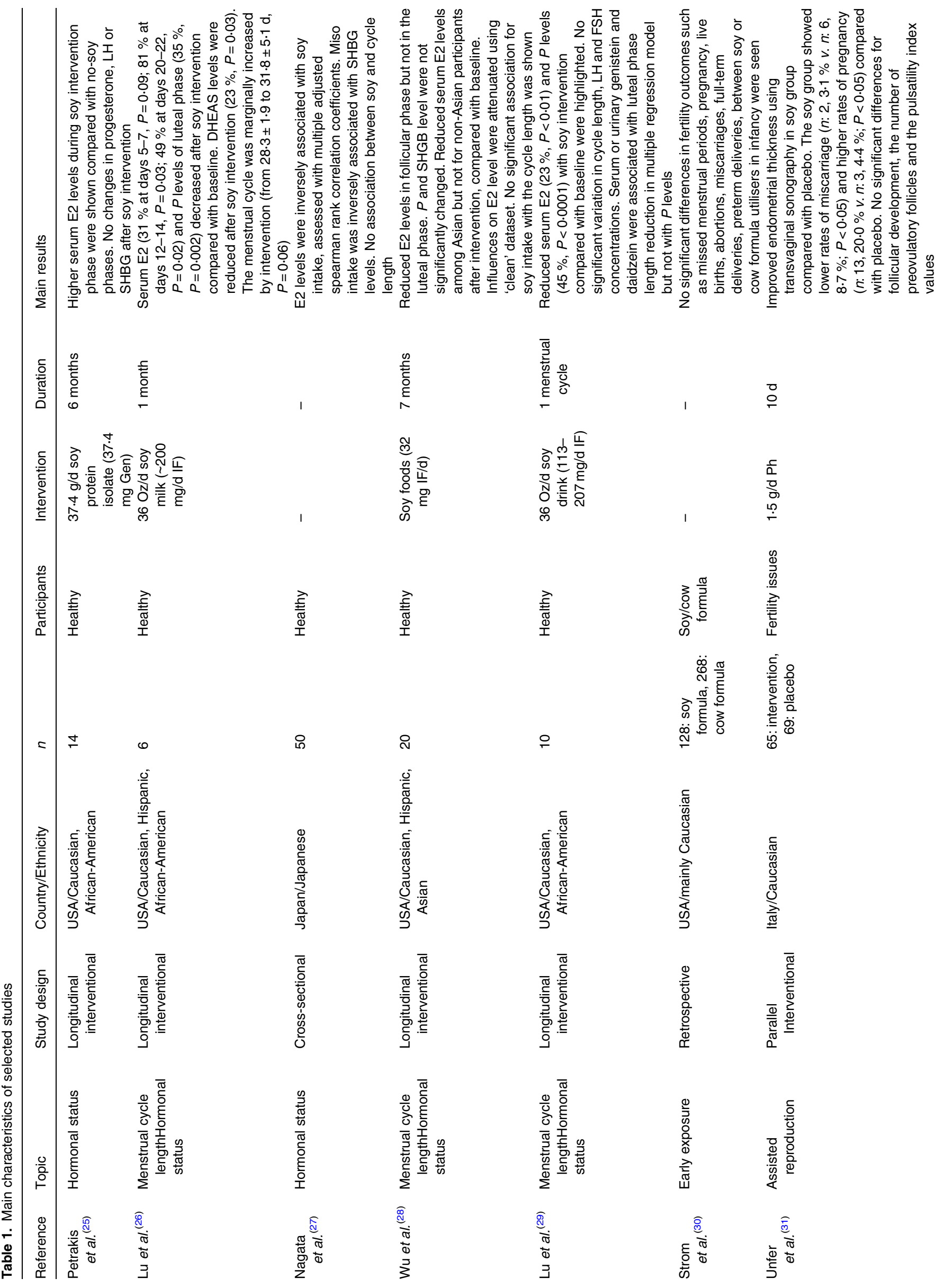



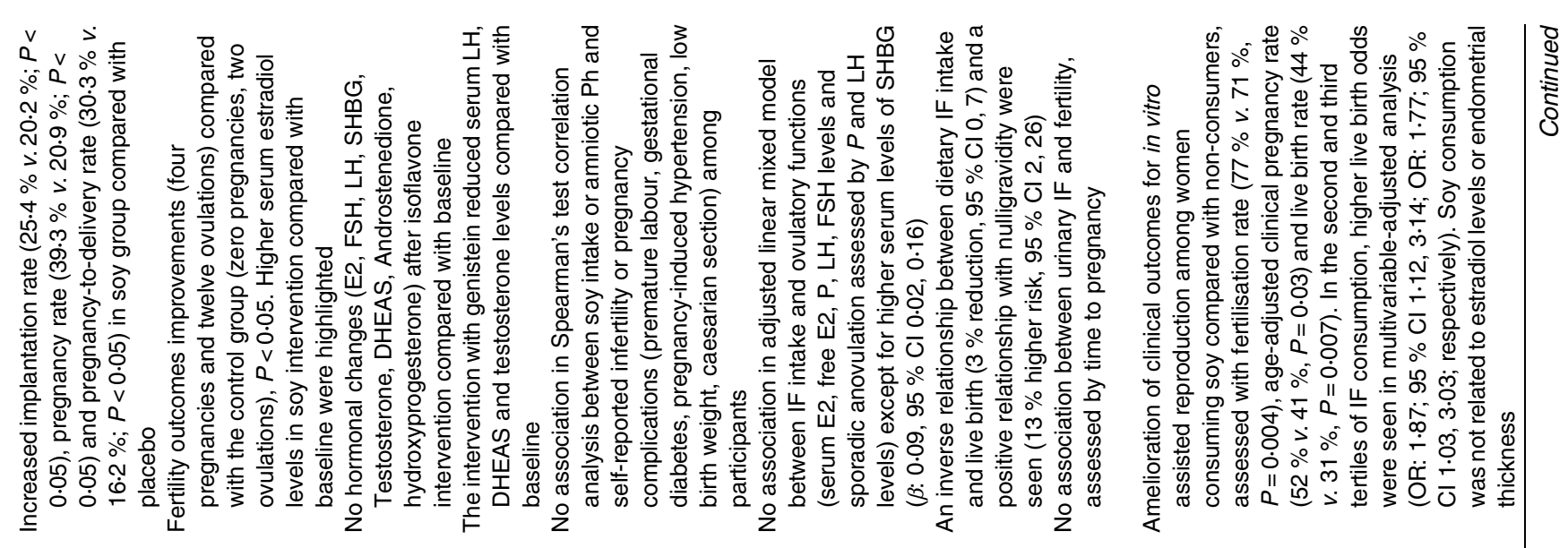

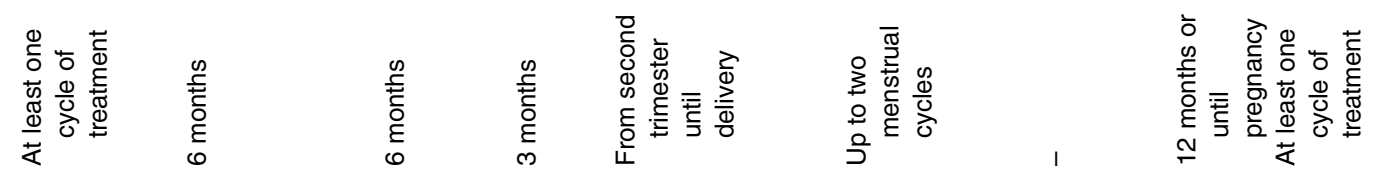

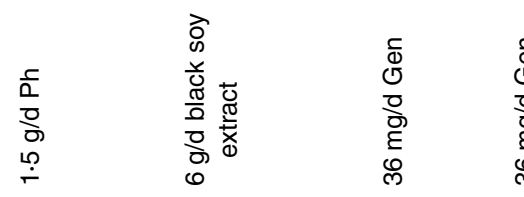

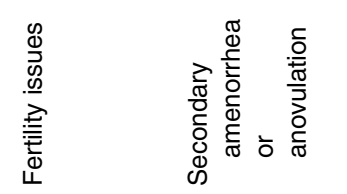

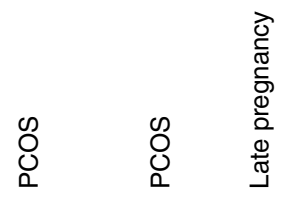

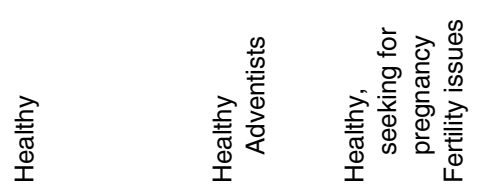

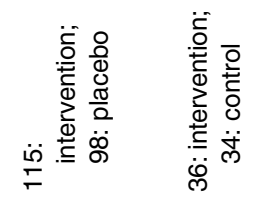

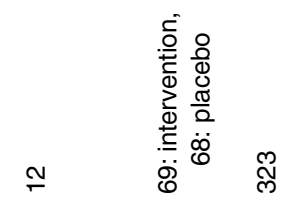

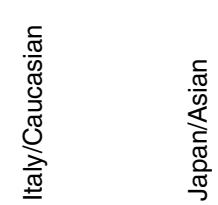

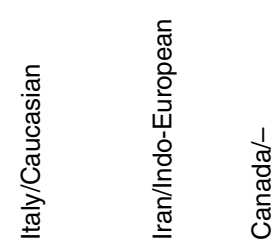

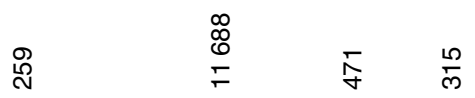

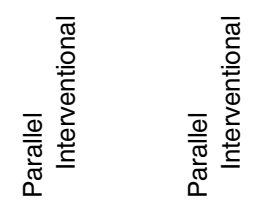
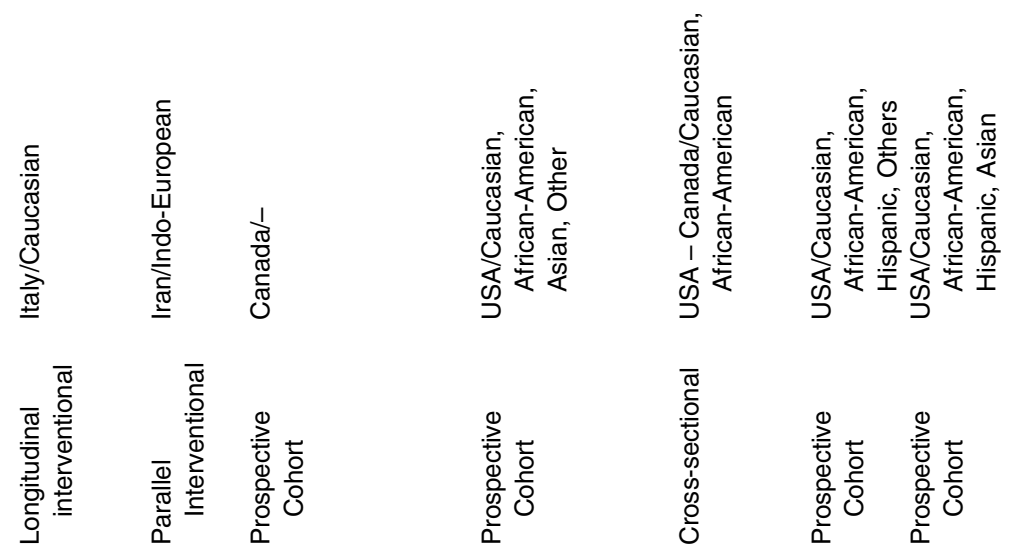

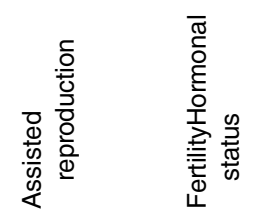

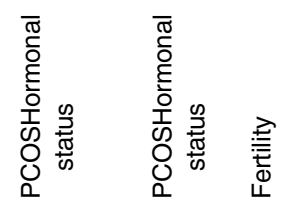

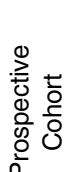

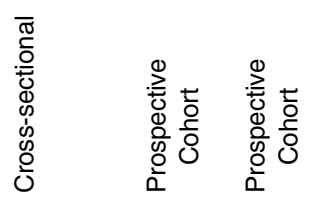

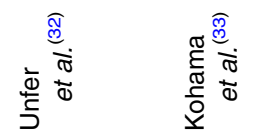

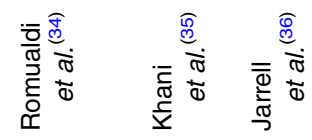

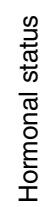

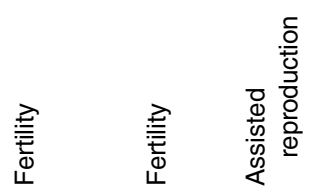

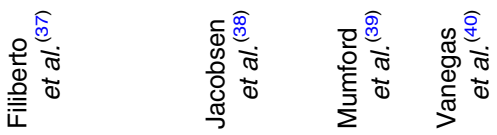




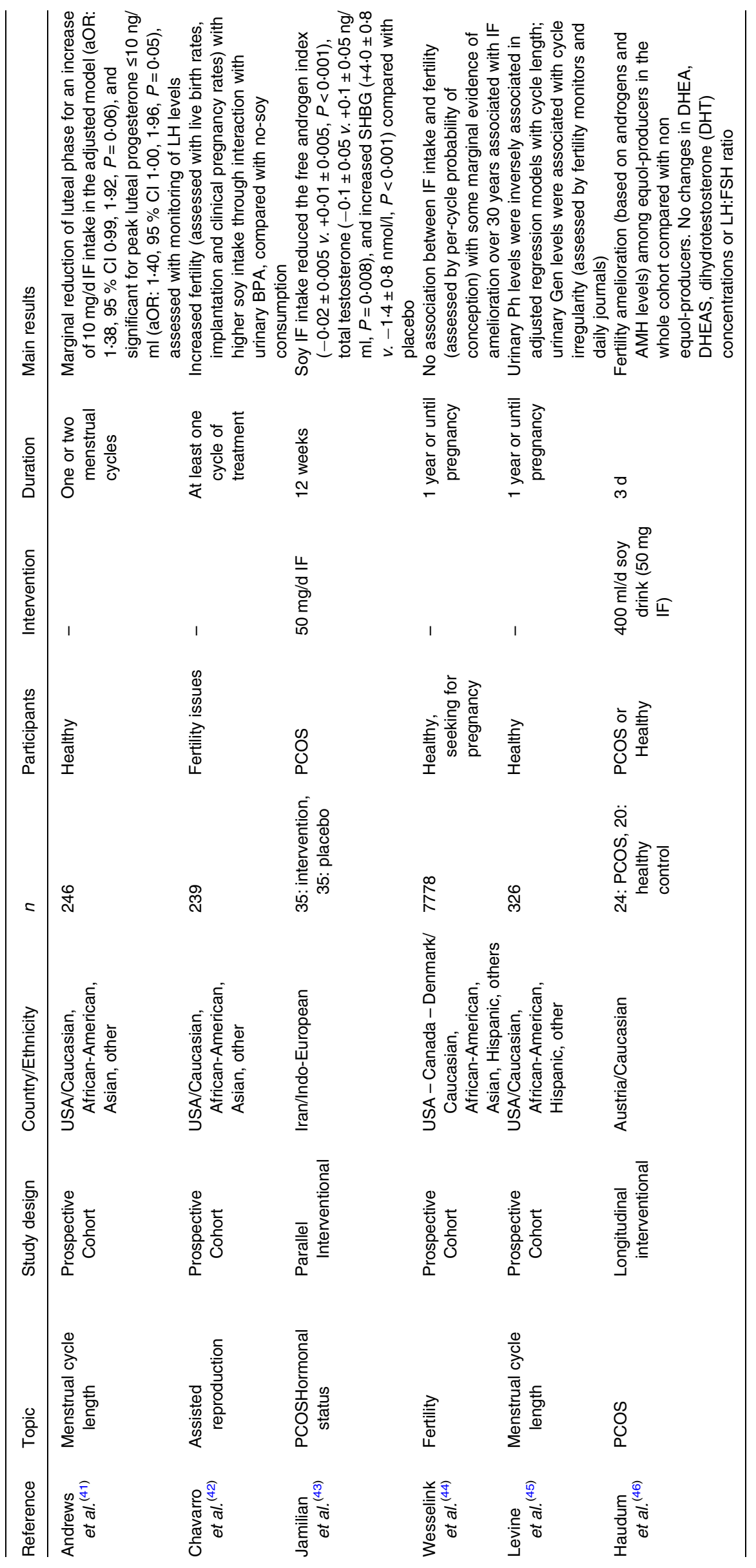


$(P<0 \cdot 05)$. Conversely, the improvements in ovulation were seen only in two patients from the control group. After 6 months, estradiol levels of patients in the intervention group were higher compared with basal $(P<0 \cdot 05)$, whereas luteinizing hormone $(\mathrm{LH})$ and follicle-stimulating hormone (FSH) were unchanged.

Black soy, in addition to the content of isoflavones, is known to be rich in antioxidant substances, especially in external seed integuments, which are rich in anthocyanins ${ }^{(49)}$. These substances could play a role in the ovaries circulatory functions ${ }^{(50)}$. Unfortunately, the work of Kohama et al. was a pilot study without a characterisation of diet among participants and without data on soy composition (isoflavone or antioxidant contents). It does not appear to be randomised and blinded, but the nature of outcomes should not be affected by these limitations.

In addition to the interventional study by Kohama and colleagues, we found three longitudinal cohort observational studies ${ }^{(36,39,44)}$ and a cross-sectional study ${ }^{(38)}$ that investigated the association between soy and fertility. Jacobsen and colleagues included 11688 American women aged 30-50 years of age who participated in Adventist Health Study-2 (AHS-2) ${ }^{(38)}$. The Adventist Church is a community with very homogeneous habits and a high prevalence of vegetarians (54\% lacto-ovo vegetarian and $7 \%$ vegan from this study $)^{(51,52)}$.

Based on this cross-sectional study, high consumption of soy isoflavones was identified ( $94 \%$ of participants). The significant inverse association between dietary isoflavone intake and live births ( $3 \%$ reduction, $95 \% \mathrm{CI} 0,7, P=0.05)$ was highlighted. Similarly, a significant correlation between isoflavone intake and nulligravidity emerged $(P=0 \cdot 03)$ with a 13

$\%$ higher risk but with a wide range of confidence interval (95\% CI 2, 26) in women with intake $\geq 40 \mathrm{mg} / \mathrm{d}$ compared with lower intakes $(<10 \mathrm{mg} / \mathrm{d})$. Only $6 \%$ of participants had not soy isoflavone intake. There was no relationship between isoflavone intake and reported problems becoming pregnant. The generalisation of these results is complex due to the type of study, which does not allow to define a causal relationship. The consumption of soy over time, the possible use of certain foods considered healthy in seeking pregnancy or the willingness to avoid pregnancy could generate spurious associations.

The use of surveys only through self-administered questionnaires, although validated, is easily exposed to self-reporting errors or incompleteness and misclassifications derived from the database used for food intake quantification. Furthermore, the nutritional habits of Adventists differ from the Western population ones and they show soy consumption more similar to populations in South-East Asia ${ }^{(53)}$.

Among selected prospective cohort studies, in 2012 Jarrell and colleagues conducted an observational study of 323 Canadian women with late pregnancy (aged at least 35) followed from the second month of pregnancy until delivery ${ }^{(36)}$. The concentration of isoflavones in the amniotic fluid was related to soy intake, but there was no significant association between soy intake or phytoestrogens in the amniotic fluid and complications of pregnancy or previous infertility.
Higher soy products intake did not correlate with the rate of infertility. However, only 106 individuals provided information on soy intake. Furthermore, the type of dietary survey carried out in the proposed environmental questionnaire was not clear. The same authors admitted that they had no information on the type of soy used and about the last ingestion. It would have been useful to have retrospective information on soy consumption to assess the potential effect on previous fertility problems. These aspects were poorly characterised by self-reporting of the participants. Other weak aspects of the population sample characterisation are given by a lack of stratification by ethnicity and equol-producers.

In another prospective study, 471 healthy American women were followed for 12 months or until delivery without showing significant correlations between urinary isoflavones, quantified by HPLC-MS analysis, and fertility, defined with adjusted Cox Model using time-to-pregnancy assessment ${ }^{(39)}$, while lignan concentrations in urine were significantly associated with shorter time to pregnancy. The study involved a large number of couples seeking pregnancy. In order to assess the association between urinary isoflavones and fertility, adjustment for various confounding factors including ethnicity, supplement use, nutrients and lifestyle aspects was applied. Measurement of urinary isoflavones and their metabolites appears to be a more reliable approach than dietary assessment alone. However, the intake of isoflavones in diet has not been investigated, and therefore, it was not possible to define the presence of equol-producers among participants. Moreover, urinary concentrations seem to reflect the isoflavone intakes in a short time window. The present study used a communitybased approach with recruitment of couples seeking pregnancy. This could have introduced other confounding factors such as the influence of male on couple's fertility or possible changes in habits caused by the desire to conceive.

Recently, Wesselink and colleagues evaluated the fertility of 7778 healthy American or Canadian women in two cohort studies that followed participants for 12 months or until pregnancy ${ }^{(44)}$. The dietary intake of isoflavones did not appear to be associated with fertility in the two cohorts but some marginal evidence of amelioration of fertility was related to a higher intake of isoflavones among $\geq 30$ years old individuals after age stratification (Fecundability Ratios: 1.12, $95 \%$ CI 9.94, 1.34 and $1.19,95 \% \mathrm{CI} 0.92,1.55$ in the two cohorts comparing $\geq 90$ th with $<24$ th percentile). There was no dose-response relation in either cohort. Correction for covariates included demographics, education, income, lifestyle, dietary and behavioural factors. However, the intakes of isoflavones in the studied cohorts were limited (range: $0 \cdot 3$ $3 \cdot 1 \mathrm{mg} / \mathrm{d}$ ). The FFQ was not specifically designed for phytoestrogen assessment and this may have underestimated intakes. Participants recruited were seeking for pregnancy and this could have been a source of confounders.

From the data obtained, diet isoflavones do not seem to have a direct effect on fertility, whether positive or negative. In the only clinical trial available, even if it is considered a pilot study, it emerges that a significant role could be played not only by isoflavones, but also by phytochemicals present in soy, particularly in black soy. This phenomenon highlights 
how in literature there is greater attention to phytoestrogens and their effect, frequently underestimating the role of other components that have a marginal interest.

\section{Menstrual cycle length in healthy women}

The length of menstrual cycle may represent an indirect marker of ovarian function and reproductive health ${ }^{(54,55)}$. Fertility is closely associated with menstrual cycle functions and a longer time to pregnancy is associated with shorter menstrual cycles ${ }^{(56-58)}$.

Five studies exploring the relationship between soy and the length of menstrual cycle in healthy women have been selected, including two observational studies ${ }^{(41,45)}$ and three longitudinal interventional studies ${ }^{(26,28,29)}$.

With regards to available clinical trials, $\mathrm{Lu}$ and colleagues conducted two interventional studies using $36 \mathrm{Oz}$ of soy milk (about one litre) divided into three daily intakes for a total daily intake of about $200 \mathrm{mg}$ of isoflavones ${ }^{(26,29)}$. In the first study, the authors administered soy milk to six American women aged 22-29 for 1 month, comparing outcomes with baseline ${ }^{(26)}$. After the soy intervention, the length of menstrual cycle marginally increased (from $28 \cdot 3 \pm 1.9$ to $31 \cdot 8 \pm 5 \cdot 1 \mathrm{~d}, P=0 \cdot 06)$. The small number of participants significantly limited the quality of results. Furthermore, there was no characterisation of dietary regimen, although it was a standard hospital diet. Participants were classified by ethnicity; however, the population sample size did not allow to perform stratification of outcomes based on this aspect. No investigation into the individual's ability to absorb and use isoflavones from soy milk was performed. Besides, the lack of a placebo group warrants caution.

In the second study by $\mathrm{Lu}$ and colleagues ${ }^{(29)}$, ten American women aged 23-42 who did not consume soy regularly were followed for the duration of a menstrual cycle, during which nutritional intervention with soy was performed $(36 \mathrm{Oz} / \mathrm{d}$ soy drink; 113-207 mg/d IF), without observing significant changes in cycle length compared to baseline and with a marginal shortening of luteal phase $(6 \%, P=0.07)$. However, in multiple regression analysis, this reduction seemed to be significantly associated with the intake of genistein and daidzein or their concentration in urine. Similar to the previous trial, the number of participants was limited. The intervention period was extended only to one menstrual cycle. Furthermore, no characterisation was made on the possible presence of equol-producers among the participants. However, the sampling during the various days of the cycle allowed a detailed characterisation of serum LH surge day. Multiple regression analysis including various set of possible confounders highlighted more in-depth correlations. The evaluation of isoflavones circulating levels and their urinary excretion allowed to show a wide inter-individual variation of metabolic and absorption capacity.

In 2000, Wu et al. conducted a 7-month interventional study on twenty healthy American women aged 21-44, half of them of Asian origin, using various types of soy foods (soy milk, edamame, tofu) for an overall daily intake of about $32 \mathrm{mg}$ of isoflavones ${ }^{(28)}$. Consistent with the previously cited data, no significant alteration in the cycle length was found among participants following the intervention. Additional considerations regarding hormonal influences will be discussed in the next paragraph.

Regarding observational studies, in 2015 Andrews and colleagues conducted a prospective cohort study on 246 American women with normal menstrual cycle, aged 18-44 and with $13 \%$ of participants of Asian ethnicity, for a follow-up of 1-2 whole menstrual cycles ${ }^{(41)}$. The authors highlighted a marginal reduction of luteal phase in the adjusted multivariable model for an increase of $10 \mathrm{mg} / \mathrm{d}$ of dietary isoflavones (aOR: 1.38, $95 \%$ CI 0.99, 1.92, $P=0 \cdot 06$ ), identified by monitoring LH levels in urine by a fertility monitor and 4-d per cycle 24 -h dietary recalls. Similar significant association was observed for peak luteal progesterone $\leq 10 \mathrm{ng} / \mathrm{ml}$ (aOR: $1 \cdot 40,95 \%$ CI $1 \cdot 00,1 \cdot 96, P=0 \cdot 05)$. Corrections for confounding factors, such as diet, demographics, lifestyle factors, age, body composition and ethnicity, indicated reliable analysis. However, the evaluation of ability to absorb and metabolise isoflavones was lacking in the present study. This is justified by the fact that the study was not designed for the specific assessment of dietary soy concerning fertility-related outcomes. Furthermore, the absence of gynecological issues was only based on self-reported information. The study must be considered exploratory, because of the limited number of luteal phase deficiency cycles and a small number of fertility-related outcomes.

Recently, in a prospective study by Levine et al., 326 American women eumenorrheic aged 18-40 were followed for 12 months or until pregnancy ${ }^{(45)}$. The authors showed an inverse correlation between cycle length (detected via fertility monitors and daily journals) and total urinary phytoestrogen levels $(-0.042 \mathrm{~d}$ for $10 \%$ increase, $95 \%$ CI -0.080 , $-0 \cdot 003)$. These also included non-soy derived phytoestrogens, such as lignans. No correlation with specific isoflavones such as equol, daidzein and O-DMA was found. Such shorter menstrual cycle length seemed not clinically relevant because shorter than $1 \mathrm{~d}$. Furthermore, for each $1 \mathrm{nmol} / \mathrm{l}$ increase of genistein, the risk of menstrual cycle irregularities increased (OR: $1 \cdot 19,95 \% \mathrm{CI} 1 \cdot 02,1 \cdot 38)$. The use of urinary phytoestrogens and their metabolites is a more reliable system compared to the evaluation of dietary intake. However, urinary phytoestrogen levels were only detected at baseline and this increased the correlation uncertainty. Despite adjustments for demographic, lifestyle, dietary factors, including ethnicity and other phytoestrogens, it would have been useful to check the dietary intake of isoflavones for equol-producers evaluation. Furthermore, the individuals recruited were seeking for a pregnancy and this could have changed their behaviour. Additionally, the enrolment criteria included only women who had stopped oral contraception less than 2 months earlier, so highly fertile individuals could have been excluded.

Luteal phase deficiency can represent a relevant aspect for pregnancy outcomes and fertility disorders. However, the association between soy and isoflavones with the reduction of luteal phase seems weak. The possible correlation between menstrual cycle length and soy does not seem convincing either. 
In the meta-analysis by Hooper and colleagues ${ }^{(59)}$ from the evaluation of eleven studies on premenopausal women, ten studies were included to clarify the effect of soy on menstrual cycle length. A slight increase of approximately $1 \mathrm{~d}(\mathrm{MD}: 1 \cdot 05$, $95 \%$ CI $0.13,1.97$ ) was seen compared with the control, with no significant effects in the length of luteal and follicular phases. However, after removing data from studies with elevated bias risk, three studies were included in the sensitivity analysis with consequent loss of statistical significance.

Emerged clinical trials display several limitations including small sample size as well as the longitudinal design without a parallel control group, placebo or a cross-over design consistently limiting the strength of these pilot studies. Similarly, the duration of interventions is limited and equol-producers have not been identified. The two observational studies also show different limitations, in particular, one of these studies uses a follow-up of only 2 months. Pending further confirmation, soy and its components do not appear to have a clinically relevant influence on menstrual cycle in healthy women.

\section{Hormonal status in healthy women}

Among the studies discussed to evaluate menstrual cycle length, three interventional studies also evaluated the levels of circulating hormones following soy intake in healthy women ${ }^{(26,28,29)}$. In the first of the two papers by $\mathrm{Lu}$ and colleagues ${ }^{(26)}$, the intake of $36 \mathrm{Oz} / \mathrm{d}$ of soy milk $(\sim 200 \mathrm{mg} / \mathrm{d}$ IF) for 1 month caused a reduction in mean estradiol levels of $31 \%$ at days $5-7, P=0 \cdot 09 ; 81 \%$ at days $12-14, P=$ $0 \cdot 03 ; 49 \%$ at days $20-22, P=0.02$, compared with the baseline.

This effect persisted for at least one menstrual cycle after the suspension of soy intake, with a maximum of persistence for three menstrual cycles. Furthermore, from the multiple regression analysis of ten women in the second trial ${ }^{(29)}$, the reduction of estradiol in both luteal and follicular phases was positively associated with serum and urinary isoflavone levels but not with individual changes in the intake. In both studies, the lowering of progesterone levels in luteal phase was also significant in the case of soy intake, mean $35 \%(P=0.002)$ compared with baseline.

Among the six women in the first clinical trial ${ }^{(26)}$, the intervention with soy also led to a significant reduction in dehydroepiandrosterone sulphate (DHEAS) levels $(23 \%, P=$ $0.03)$, an intermediary in estradiol synthesis. In the ten women who participated in the second study ${ }^{(29)}$, there were no significant changes in the levels of luteinizing and follicle-stimulating hormones. The lack of variation in gonadotropins can explain the absence of variation in menstrual cycle. However, soy diet reduced progesterone $(45 \%, P<0.0001)$ and estradiol levels $(23 \%, P<0 \cdot 01)$, compared with baseline. The urinary or serum levels of isoflavones did not affected progesterone levels in the multiple regression analysis. The strength of these studies was the assessment of hormone levels based on the menstrual cycle phase. The limitations of these studies have already been discussed in the previous paragraph.

In another clinical trial already discussed, although no changes in cycle length were found following soy foods intervention in twenty women with a follow-up of at least seven menstrual cycles, a significant reduction in follicular phase by $9.3 \%(P<0.05)$ in estradiol concentrations was observed, but not in luteal phase ${ }^{(28)}$. Progesterone and sex hormone-binding globulin (SHGB) levels were not significantly changed by soy intake. The reduction of estradiol concentrations observed became statistical marginal $(8.9 \%, P=$ $0 \cdot 06)$ when analysis was restricted to the 'clean' dataset: data after exclusion of thirteen specimens collected too soon or too late after ovulation. From a sub-analysis on ethnic characteristics, it was further highlighted that only Asian women showed a significant reduction in follicular estradiol from baseline $(17 \cdot 4 \%)$.

From the analysis of urinary excretion of isoflavones normalised for creatinine during the intervention with soy, Asian women had significantly greater excretion of isoflavones than non-Asian women. However, the difference became not significant after adjustment for isoflavone intake. Isoflavones concentrations did not show significant differences between participants at baseline.

The clinical trial was limited to a small sample size, lacking of control/placebo group and there was no characterisation of equol-competence. However, the work had several strengths: the real evaluation of luteal and follicular phase through the dosage of urinary LH:creatinine ratio, the characterisation of sampling according to the cycle; the evaluation of isoflavone content in foods used for the intervention and quantification of urinary isoflavones to check compliance; the use of soy foods and not supplements or extracts to approach a real-life pattern; the characterisation of diet at various steps of clinical trial to avoid confounding mechanisms; the stratification by ethnicity which indirectly showed the effect on equol-producer individuals.

This latter aspect suggests a differential capacity for metabolising isoflavones even if these differences were no longer significant when corrected for the intake of isoflavones and estradiol levels were not significantly associated with urinary excretion of isoflavones. In addition, no significant changes in progesterone, LH or SHBG were found in the whole study sample. From the sub-analysis by ethnic stratification, follicular SHBG levels were higher in non-Asians.

Previously, Petrakis and colleagues proposed an interventional study with a soy isolate $(37.4 \mathrm{~g}$ of soy protein containing $37.4 \mathrm{mg}$ of genistein) on twenty-four women (pre- and postmenopause) followed for 6 months plus 3 months preintervention and 3 months post-washout ${ }^{(25)}$. Estradiol levels showed increased plasma concentrations during the intervention period among premenopausal women $(n: 14)$ in both luteal and follicular phases (composite menstrual cycle assessment). No changes in progesterone and SHBG concentrations from baseline were observed. Compliance with the intervention was suggested by urinary excretion of isoflavones. While isoflavones and their metabolites were undetectable in the presoy phase, during intervention the $24 \mathrm{~h}$ output of urinary excretion was $3.12 \mathrm{mg}$ for genistein $(7.4 \%$ of the ingestion). Excretion of daidzein and its metabolites dihydrodaidzein and $O$-desmethylangolensin $(36 \cdot 01,3 \cdot 14$ and $2.27 \mathrm{mg}$, respectively) accounted for $42.1 \%$ of daidzein ingested. Although 
this clinical trial showed the long-term effect of soy ingestion on serum hormone levels, it was a pilot study with a limited number of participants (fourteen premenopausal women). Furthermore, diet and energy intake were not investigated and sampling was not well-timed to menstrual cycle. They evaluated the hormonal variations during menstrual cycle through the 'composite' construct that considered the cumulative information of the day of menstrual cycle for specimens. The authors defined the unusual estradiol increase as 'erratic'.

Based on our literature search, we also identified two observational studies: a cross-sectional study published in 1997 by Nagata et al. ${ }^{(27)}$ and a longitudinal study published in 2013 by Filiberto et al. ${ }^{(37)}$. In the study by Nagata and colleagues, fifty Japanese women were enrolled to evaluate the association between soy intake (using an FFQ) and hormone levels. After adjustments, an inverse correlation between estradiol and soy intake was highlighted on the 22nd day of menstrual cycle (r: $-0 \cdot 32, P=0 \cdot 04)$ but not on the 11 th.

SHBG levels were not associated with the intake of soy foods, except in the case of miso intake on the 22nd day of cycle $(r:-0 \cdot 36, P=0 \cdot 02)$. Day 22 should correspond to the mid-luteal phase, however, the authors pointed out that participants exhibited different lengths of menstrual cycle and this could have been a source of heterogeneity that was used as a covariate in the regression model. However, soy intake did not correlate with cycle length $(r:-0 \cdot 12, P=0 \cdot 45)$. The evaluation at two different times of menstrual cycle allowed to discriminate the effect between luteal and follicular phases but not day by day hormonal fluctuations. However, the number of participants was limited for a cross-sectional study, and dietary survey through frequency questionnaires in the absence of an assessment of blood or urine isoflavone levels could lead to uncertainty. The study's strength include a large consumption of soy and by consistent inter-individual variability among participants (total intake of $37.9 \pm 26 \cdot 1 \mathrm{~g} / \mathrm{d}$ ), which allows better detection of cross-sectional correlations.

Instead, in the cohort study by Filiberto and colleagues, 259 American women were followed for at least 2 menstrual cycles. Mildly increased levels of SHBG were associated with higher dietary isoflavone intakes (Q4 $[1 \cdot 6-78 \cdot 8 \mathrm{mg} / \mathrm{d}] v$. Q1 $[0 \cdot 0-$ $0.3 \mathrm{mg} / \mathrm{d}])$ in the adjusted linear mixed model $(\beta: 0.09,95$ $\%$ CI $0 \cdot 02,0 \cdot 16$ ), but no correlation was found for estradiol, progesterone, LH, FSH levels or anovulatory events. $44 \%$ of women of Asian descent were in the highest quartile of isoflavone intake. Although isoflavones can be found in many foods, not soy foods can be considered negligible sources of these compounds. The influence on SHBG levels can have a beneficial effect from an endocrine point of view, without negative effects on ovulation. The present study has numerous strengths: a large sample of participants with good adherence to the study, a detailed assessment of dietary habits, and comprehensive sampling during all phases of menstrual cycle. Finally, the authors made a detailed assessment of confounders (diet, ethnicity, age and BMI). However, there were also limitations: the duration of the study which was limited to two menstrual cycles and an evaluation of equol-producers among individuals was lacking. Furthermore, the intake of isoflavones among participants was very low and this made it difficult to compare the findings with clinical trials that often use intakes similar to Asian populations (23-84.4 mg/d).

In the previously mentioned meta-analysis by Hooper and colleagues $^{(59)}$, reduction of about $22 \%$ of FSH (SMD: $-0.45 \mathrm{UI} / 1,95 \% \mathrm{CI}-0.79,-0.11, P=0.01)$ and of about $4 \%$ of $\mathrm{LH}$ (SMD: $-0.34 \mathrm{IU} / 1,95 \%$ CI $-0 \cdot 68,-0 \cdot 01$, $P=0 \cdot 05)$ were related to the intake of soy or isoflavones. However, levels of progesterone, estradiol, free estradiol, estrone and SHBG did not show significant differences.

These changes may have resulted in the mild, non-clinically relevant prolongation of menstrual cycle, as discussed in the previous section. However, after removing data from studies with elevated bias risk, two studies were included in the sensitivity analysis with a consequent loss of statistical significance for LH levels. Nevertheless, a reduction in FSH levels was confirmed (SMD: $-0.87 \mathrm{IU} / 1,95 \%$ CI -1.72, -0.02). However, the number of combined participants of the two studies was very limited ( $n: 40)$.

Steroid hormones (estradiol, progesterone and DHEAS) play a role in epithelial cell proliferation in mammals. The duration of menstrual cycle, especially in luteal phase, can also have a direct influence on the mammary gland proliferation, through a reduction in exposure of the epithelium to proliferative hormones. For these reasons, studies that evaluated the ovarian hormones secretion were aimed at exploring the potential beneficial effect of soy on breast cancer prevention, but they were not designed for the evaluation of endocrine consequences, including fertility.

The reduction of estradiol and progesterone could postpone ovulation by lengthening the menstrual cycle. The effects obtained from selected studies do not seem to show a clear significance regarding fertility and menstrual cycle length, as discussed in the previous paragraph. There is a limited trend in estradiol reduction related to soy consumption; however, in their interventional study, Petrakis and colleagues observed an unusual increase of estradiol levels ${ }^{(25)}$.

In addition, in the work of Kohama and colleagues, an increase in estradiol levels following intervention with soy compared with baseline was shown ${ }^{(33)}$. However, the subjects enrolled were women with secondary amenorrhea and therefore this variation could have a different meaning compared with results discussed in this section, obtained in the healthy population.

There are clues about the association between soy intake and the increase in SHBG levels. It is plausible that isoflavones bind to this blood carrier and stimulate its hepatic synthesis. This could favour the bioavailability of sex hormones ${ }^{(60)}$. However, in the work of Filiberto and colleagues ${ }^{(37)}$, even if the correlation between isoflavones and the increase in SHBG was highlighted, the dosage of estradiol and free estradiol did not show significant correlations, although the estimate of free estradiol was done through Sodergard's formula ${ }^{(61)}$, so a direct dosage would be more reliable.

It is important to evaluate the levels of hormones that fluctuate during the cycle at several points. This could be done by empirically monitoring ovulation to get a real information of menstrual phase, such as quantifying the urinary LH peak as a marker of ovulation, as done by Wu et al. ${ }^{(28)}$. 


\section{Polycystic ovary syndrome}

Polycystic ovary syndrome (PCOS) is a major endocrine and metabolic disorder in women ${ }^{(62,63)}$. It is an endocrine dysfunction that includes hormonal alterations (increased levels of adrenal and ovarian androgens and SHBG secretion from the liver) and anovulatory disorders ${ }^{(64)}$. These alterations easily lead to hyperandrogenism and irregular menstrual cycles. Furthermore, women with PCOS display a higher prevalence of hyperinsulinemia, dyslipidemia, insulin resistance and obesity compared to healthy population. The disease etiology is still debated but it seems to involve inflammatory mechanisms and oxidative stress ${ }^{(65,66)}$. The diagnosis of PCOS occurs in the presence of at least two of the three Rotterdam Criteria: oligo or anovulation, polycystic ovary morphology and biochemical or clinical hyperandrogenism ${ }^{(67)}$.

Four clinical trials were found among search engines results: two longitudinal pilot studies $^{(34,46)}$ and two interventional studies with a parallel design, both conducted in Iranian populations $^{(35,43)}$.

Romualdi and colleagues in 2008 enrolled twelve Italian women with metabolic syndrome and PCOS and with a follow-up of 6 months using $36 \mathrm{mg} / \mathrm{d}$ of oral genistein as an intervention ${ }^{(34)}$. Researchers did not observe any clinical improvement, alteration of menstrual cycle or hormonal alteration (estradiol, SHBG, DHEAS, androstenedione, testosterone, FSH, LH) compared with baseline levels. Patients showed plasma androgens levels above or at the upper limit the normal range, at baseline. Improvements were observed only in lipid profile (circulating total cholesterol, LDL and LDL/HDL ratio and triglycerides). Despite the 6-month duration of the clinical trial, the lack of a placebo group, the absence of characterisation of equol-competence among individuals and the limited number of participants reduced the strength of the results obtained.

The same amount of genistein was used in a parallel clinical trial on 137 Iranian women with PCOS with a 3-month follow-up ${ }^{(35)}$. In the present study, the intervention group showed improvements in hormonal circulating levels compared with baseline, which consisted in the reduction of $\mathrm{LH}$ levels $(-9.4 \%, P=0.000)$, testosterone $(-5.6 \%, P=0.000)$ and DHEAS $(-8.7 \%, P=0 \cdot 000)$, with no significant changes in the control group. FSH levels were not significantly changed after genistein intervention. Genistein treatment reduced LDL cholesterol and triglycerides levels. Sampling involved synchronisation on the third day of menstrual cycle follicular phase, spontaneous or pharmacologically induced. Although the clinical trial was quasi-randomised, with a placebo group, double-blinded, authors did not characterise the dietary regimen of individuals as well as their ability to effectively absorb and metabolise soy isoflavones. Furthermore, hormone levels were evaluated only at baseline, without taking into account the differences between the two groups.

Jamilian and colleagues in 2016 conducted another parallel clinical trial on seventy Iranian women with PCOS, using $50 \mathrm{mg} / \mathrm{d}$ of soy isoflavones for a 1-month follow-up ${ }^{(43)}$. From the general linear model of the analysis of covariance, the intervention with soy reduced free androgen index
$(-0.02 \pm 0.005 v \cdot+0 \cdot 01 \pm 0 \cdot 005, P<0 \cdot 001)$, total testosterone $(-0.1 \pm 0.05 \quad v . \quad+0.1 \pm 0.05 \mathrm{ng} / \mathrm{ml}, P=0.008)$ and increased SHBG levels $(+4 \cdot 0 \pm 0 \cdot 8 \quad v \cdot-1 \cdot 4 \pm 0 \cdot 8 \mathrm{nmol} / \mathrm{l}$, $P<0.001$ ) compared with placebo (adjusted for baseline values). No significant differences were appreciated for free testosterone and DHEAS. The hormonal improvement has been followed by clinical ameliorations such as the reduction of alopecia, serum insulin levels, HOMA-B (homeostasis model of assessment-B cell function) and HOMA-IR (homeostasis model of assessment-insulin resistance) index among patients in the intervention arm. Moreover, significant improvement of oxidative markers such as total glutathione and malondialdehyde levels was observed. Although this was a randomised, placebo-controlled and double-blinded trial with a sample size appropriate to the power of detection, there was no evaluation of serum and urinary levels of isoflavones and/or metabolites. There was no evaluation of dietary habits and the determination of hormone levels was performed using non-validated ELISA kits, due to limited budget. For these reasons, results should be interpreted with caution.

Recently, Haudum and colleagues conducted a longitudinal case-control clinical trial on forty-four Australian patients (twenty-four PCOS and twenty healthy controls) using 400 $\mathrm{ml} / \mathrm{d}$ of soy milk (containing approximately $50 \mathrm{mg}$ of isoflavones, $13.2 \mathrm{~g}$ protein) for a 3 -d pilot study ${ }^{(46)}$. The study included the evaluation of patients' microbiota composition as the primary endpoint, but androgen levels were also evaluated with $\mathrm{AMH}$ as markers of fertility as a secondary endpoint.

Major equol production was associated with a reduction in androgens levels (total testosterone, free testosterone and androstenedione), in the whole cohort. However, stratification for the control group or PCOS patients did not show a significant correlation between androgens and equol production. Likewise, equol-producers showed lower AMH levels in the whole cohort as well as in participants in PCOS or control groups. No changes were highlighted for DHEA, DHEAS, dihydrotestosterone (DHT) concentration or LH:FSH ratio. Microbial alpha diversity and glucose homeostasis improved in PCOS group after isoflavone intervention, resembling the control group profile at baseline. This was a short pilot study with a small sample size in subgroups. The power analysis concerning variation in isoflavone urinary excretion accounted for a sample size of 25 for $>90 \%$ detection power. Furthermore, the use of spot urine samples could generate an underestimation of urinary isoflavones quantification. Even if the clinical trial did not include a placebo group or randomisation, the presence of a control group and the evaluation of equol-producer individuals mitigated these issues.

From data that emerged on individuals with PCOS, there is no homogeneous improvement effect on hormonal picture, on menstrual cycle and therefore on fertility associated with soy consumption. Overall, a trend toward improvement can be appreciated but further studies are necessary to confirm the beneficial effect. Among the studies already cited, however, we must consider the work of Kohoama and colleagues ${ }^{(33)}$, which showed fertility improvements following intervention with black soy extract in individuals with secondary amenorrhea, including patients with PCOS. 


\section{Assisted reproduction technology}

Interestingly, soy often appears in literature as a food with a beneficial effect on fertility, especially in the case of pregnancy search $^{(68)}$. Four papers were found about assisted reproduction technology, two of which were interventional studies by Unifer and colleagues, using high intakes of soy phytoestrogens as adjuvant ${ }^{(31,32)}$. Moreover, two recent observational cohort studies by Chavarro and colleagues evaluated the association between soy consumption and in vitro fertilisation outcomes $^{(40,42)}$.

In the first clinical trial by Unifer and colleagues, $1500 \mathrm{mg} / \mathrm{d}$ of isoflavones from soy or placebo were administered for $10 \mathrm{~d}$ to 134 women who had been infertile for at least 2 years, undergoing intrauterine insemination after $100 \mathrm{mg} / \mathrm{d}$ for $5 \mathrm{~d}$ of clomiphene citrate treatments (an ovulation inducer) ${ }^{(31)}$. Despite the significant increase in FSH, LH and estradiol in both intervention arms, the endometrial thickness (assessed by transvaginal sonography) had a major improvement in the intervention group compared with placebo. Furthermore, the intervention group showed lower rates of miscarriage $(n: 2,3.1 \% v \cdot n: 6,8 \cdot 7 \% ; P<0 \cdot 05)$ and higher rates of pregnancy $(n: 13,20 \cdot 0 \% v \cdot n: 3,4.4 \% ; P<0.05)$ compared with placebo. Follicular development, the number of preovulatory follicles and the pulsatility index values were not different between groups after intervention.

Presumably, treatment with pharmacological concentrations of soy phytoestrogens allows mitigating the negative effect of clomiphene citrate on endometrial tissue, thus facilitating embryo implantation.

The same type of soy phytoestrogen intervention was subsequently used by Unifer and colleagues in a second clinical trial on 213 infertile women undergoing in vitro fertilisation with embryo transfer cycles after intramuscular progesterone treatments $(50 \mathrm{mg} / \mathrm{d}$ ) with or without (placebo) $1500 \mathrm{mg} / \mathrm{d}$ of soy isoflavones intake ${ }^{(32)}$.

The concomitant treatment with soybean phytoestrogens significantly increased the implantation rate $(25 \cdot 4 \%$ v. $20 \cdot 2$ $\% ; P<0.05)$, the pregnancy rate $(39.3 \%$ v. $20.9 \% ; P<$ $0 \cdot 05)$ and the pregnancy-to-delivery rate $(30 \cdot 3 \%$ v. $16.2 \%$; $P<0 \cdot 05)$ compared with placebo. No significant differences were found in the spontaneous abortion rate, the number and quality of embryos transferred or oocytes fertilised. Regarding the two mentioned studies, the use of very high amounts of isoflavones is noteworthy because it is not possible to obtain such a dose through diet, therefore the effects found can be interpreted as a pharmacological and not nutritional intervention.

These clinical trials had several strengths including the presence of a placebo group, randomisation, double-blinding and recruitment of a wide number of participants. Moreover, couples with male infertility issues were excluded. The influence of high-dose of isoflavones on fertility emerging from the studies is difficult to be transferred to other groups of individuals with other ethnicity or different treatments.

Regarding the observational studies available, in 2015 Venegas et al. recruited 315 USA women underwent 530 cycles of assisted reproduction technology ${ }^{(40)}$. There was a significant correlation between dietary soy consumption and fertilisation rate $(77 \% v .71 \%, P=0.004)$, age-adjusted pregnancy (52 $\% v .41 \%, P=0.03)$ or age-adjusted live birth rate $(44 \% v$. $31 \%, P=0 \cdot 007$ ) among soy consumers compared with nonconsumers. Participants were divided into four categories: non-consumers and tertiles of soy intake. Live birth odds in the multivariable-adjusted analysis was higher among women in the second tertile of soy intake, consuming $2 \cdot 64-7 \cdot 55$ $\mathrm{mg} / \mathrm{d}$ of soy isoflavones (OR: 1.87; $95 \%$ CI 1.12, 3.14) and among women in the third tertile of soy intake, consuming 7.56-27.89 mg/d of isoflavones (OR: 1.77; $95 \%$ CI 1.03, 3.03) compared with no consumption, but without a significant linear trend. The adjustment for male partner intake of soy in the subgroup analysis did not change the association. As expected, women with the highest soy consumption were more likely to be of Asian descent. Soy consumption was not related to estradiol levels or endometrial thickness. This aspect was different from the results of the clinical trials with high intakes listed above, perhaps due to very different intakes (mean isoflavone consumption of $3.4 \mathrm{mg} / \mathrm{d}$ in this cohort). Despite the sample size and full follow-up for endpoints evaluation, the study displays limitations. The ethnicity assessment of participants was useful in identifying, as might be expected, a greater consumption of soy foods by Asian individuals. However, ethnicity was not used for outcomes stratification. Furthermore, there was no evaluation of metabolic utilisation capacity of isoflavones and their absorption by measuring serum and urinary levels. This may have influenced the presence of large confidence intervals.

Similar to the previous observational study, Chavarro et al. conducted another prospective cohort study on 239 American women undergoing assisted reproductive technology ${ }^{(42)}$. The authors found an association between pregnancy outcomes and urinary Bisphenol A (BPA), dependent on soy consumption in the multivariable-adjusted mixed model. Implantation ( $P$ for interaction $<0 \cdot 02$ ), pregnancy $(P$ for interaction $<0 \cdot 03)$ and live birth rates $(P$ for interaction $<0 \cdot 01)$ were higher among soy-consumers $(n: 176,74 \%$; mean isoflavone intake of $3.4 \mathrm{mg} / \mathrm{d}$ ) without linear dependence with urinary BPA quartiles $(P$ trend $>0.05)$, compared with no consumer who had lower rates with higher BPA excretion ( $P$ trend $<0 \cdot 05)$. This suggests a protective effect of soy against fertility disturbance by BPA. The study did not evaluate circulating or urinary levels of isoflavones to verify the ability to metabolise isoflavones. Furthermore, the use of spot urine samples for BPA quantification may have underestimated its exposure.

While the observational data better reflect the effects of diet in free-living conditions compared with experimental settings of clinical trials, the use of food frequency questionnaires exposes to possible misclassification and measurement errors. Furthermore, the evaluation of dietary pattern before infertility treatments does not exclude the possibility that soy consumption may have been influenced by the search for a healthy pattern to achieve pregnancy.

From obtained data, it seems likely that soy consumption, not only in the form of isoflavones in pharmacological quantities, could have a beneficial effect on fertility, especially in 
those individuals with fertility problems. However, among fertile individuals, it may have a neutral effect, as discussed in the previous paragraphs.

\section{Influence of soy in early stages}

Although some works investigate the relationship between consumption of soy formulations in infancy and age at menarche, as well as the onset of puberty or pre-puberty reproductive organ size, these outcomes are not strictly related to fertility in reproductive age ${ }^{(69-71)}$. The possibility of a sexual development disorder as a neonatal programming effect is an often raised hypothesis because circulating levels of isoflavones are higher in soy-fed infants compared with cow milk formula or breastfed infants ${ }^{(69)}$.

However, a clear effect on reproductive system has never been highlighted, especially due to the absence of observational studies designed for this purpose. In 2015, a longitudinal study found no differences in sexual organ development at 5 years of age between cow milk formula, breast milk and soy formula feeding ${ }^{(69)}$. Although not strictly related to the aspect of fertility, the study is still ongoing (Clinicaltrials.gov: NCT00616395) intending to follow the participants to evaluate effects on reproductive functions, later in life.

The only study found about the effect of exposure to soy in childhood and reproductive functions is the retrospective study by Strom and colleagues ${ }^{(30)}$. The authors found no significant differences in reproductive outcomes (missed menstrual periods, pregnancy, live births, abortions, miscarriages, full-term deliveries, preterm deliveries, etc.) between 128 women fed with soy-based formula and 268 women fed with cow milk formula during infancy. Longer, not clinically relevant duration of menstrual bleeding (adjusted MD: 0.37 d, $95 \%$ CI $0.06,0.68)$, without differences in severity of menstrual flow was observed. However, results are questionable due to the lack of hormone level measurements or reproductive functions. Currently, data are insufficient to assess the effect of early-stage soy exposure on fertility-related outcomes.

Table 2 summarises main limitations about the studies discussed. Only three articles declared power analysis to assess adequate sample size ${ }^{(30,43,46)}$. However, this omission does not necessarily imply that the assessment has not been carried out.

\section{Proposed mechanisms of isoflavones action}

The chemical structure similarity between soy isoflavones and endogenous estrogens has always stimulated the attention for this class of compounds. However, the mechanisms underlying isoflavones effects on human health are manifold.

The interaction between isoflavones and ER $\beta$ estrogen receptor results in a competitive effect which in turn blunts endogenous estrogens action ${ }^{(72)}$, as suggested by estrogenic activity of biochanin A and genistein on BT-474 human breast cancer ${ }^{(73)}$. Isoflavones also bind to $\mathrm{ER} \alpha$ receptor, albeit with lower affinity. These mechanisms involve genomic regulation with activation of both receptor's subtypes at $1 \mu \mathrm{M}$ as seen in 293 human embryonal kidney cells in transient gene expression assay ${ }^{(74)}$.

Isoflavones also show effects that do not imply ER $\alpha$ and ER $\beta$ involvement. They can bind G-protein-coupled estrogen receptor 1 (GPR30), with effects driven by both genomic and non-genomic regulation involving different cellular signalling pathways, such as intracellular increase of calcium or NO levels ${ }^{(75)}$, as observed in human endothelial cells after stimulation with equol $100 \mathrm{nM}^{(76)}$. Isoflavone genistein inhibited the enzyme tyrosine kinase in human A431 cell membranes at $0.7 \mu \mathrm{g} / \mathrm{ml}$, a very high, non-physiological concentration ${ }^{(77)}$ and act as a ligand for peroxisome proliferator-activated receptors (PPARs) in cultured astrocytes at $500 \mathrm{nM}^{(78)}$. In addition, equol acts on incretins levels in endocrine L cell line GLUTag cells at concentration ranging from 50 to $300 \mu \mathrm{M}$, with longterm metabolic consequences ${ }^{(79)}$.

Phytoestrogens can modulate endogenous hormones at micromolar concentrations by influencing the expression of the enzymes cytochrome P450 19 aromatase (Cyp19), 17ß-hydroxysteroid

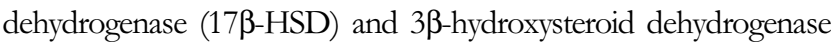
(3ß-HSD), steroid sulfatases (STS) and sulfotransferases (SULTs), enzymes of steroid biosynthetic pathway ${ }^{(80-82)}$. These enzymes convert estrone and androstenedione into estrogen and testosterone ${ }^{(83,84)}$.

Furthermore, phytoestrogens appear to act on SHBG synthesis by altering mRNA levels in hepatocarinoma human cells treated with genistein $20 \mu \mathrm{M}^{(85)}$, and modulating the balance between bound and free steroids or competing with endogenous sex hormones for the active site binding of the carrier ${ }^{(86)}$.

Last but not least, soy isoflavones can act through an antioxidant mechanism through the stimulation of enzymes responsible for xenobiotics metabolism and oxidative stress reduction in vitro at a range of $5-100 \mu \mathrm{M}^{(87)}$.

Isoflavones in human plasma are usually low $(0.4-157 \mathrm{nM})$ in individuals consuming low-isoflavone diets but in large soyconsumers, such as Asian people, isoflavone concentration can reach up to $\sim 4 \mu \mathrm{M}$, with equol reaching up to $\sim 40 \mathrm{nM}$ in low consumers and up to $\sim 2 \mu \mathrm{M}$ in large soy-consumers ${ }^{(88)}$.

Fig. 2 highlights the main cellular mechanisms attributed to isoflavones.

\section{Strengths and limitations}

Among the limitations of this review of literature, we must include the possible avoidance of studies that considered fertility as a secondary outcome and which therefore may have escaped from the manuscript selection. In addition, non-English papers could have provided relevant data on the topic, especially those from countries with a more consistent history of soy consumption. However, for articles selection, we used search engines both for scientific literature and specific for clinical trials, without filters application that could have limited the results. Furthermore, the search for sources has been extended to the single manuscripts reference lists. Notably, during manuscript preparation, Messina and colleagues published a technical review on endocrine effect of soy and isoflavones ${ }^{(90)}$. The authors of this recent literature review 
Table 2. Limitations of clinical study

\begin{tabular}{|c|c|c|c|c|c|c|}
\hline Reference & Randomisation & Blinding & Power analysis & Placebo group & Diet characterisation & Adjustment for confounders \\
\hline Petrakis et al. ${ }^{(25)}$ & No & No & No & No & No & No \\
\hline Lu et al. ${ }^{(26)}$ & No & No & No & No & No & No \\
\hline Nagata et al. ${ }^{(27)}$ & - & - & No & - & Yes & Yes \\
\hline Wu et al. ${ }^{(28)}$ & No & No & No & No & Yes & No \\
\hline Lu et al. ${ }^{(29)}$ & No & No & No & No & Yes & Yes \\
\hline Strom et al. ${ }^{(30)}$ & - & - & Yes & - & Partial & Yes \\
\hline Unfer et al. ${ }^{(31)}$ & Yes & Yes & No & Yes & No & No \\
\hline Unfer et al. ${ }^{(32)}$ & Yes & Yes & No & Yes & No & No \\
\hline Kohama et al. ${ }^{(33)}$ & No & No & No & Control & No & No \\
\hline Romualdi et al. ${ }^{(34)}$ & No & No & No & No & No & No \\
\hline Khani et al. ${ }^{(35)}$ & Partial & Yes & No & Yes & No & No \\
\hline Jarrell et al. (36) $^{2}$ & - & - & No & - & Partial & No \\
\hline Filiberto et al. $^{(37)}$ & - & - & No & - & Yes & Yes \\
\hline Jacobsen et al. ${ }^{(38)}$ & - & - & No & - & Yes & Yes \\
\hline Mumford et al. ${ }^{(39)}$ & - & - & No & - & No & Yes \\
\hline Vanegas et al. ${ }^{(40)}$ & - & - & No & - & Yes & Yes \\
\hline Andrews et al. ${ }^{(41)}$ & - & - & No & - & Yes & Yes \\
\hline Chavarro et al. ${ }^{(42)}$ & - & - & No & - & Yes & Yes \\
\hline Jamilian et al. ${ }^{(43)}$ & Yes & Yes & Yes & Yes & No & Yes \\
\hline Wesselink et al. ${ }^{(44)}$ & - & - & No & - & Partial & Yes \\
\hline Levine et al. ${ }^{(45)}$ & - & - & No & - & Partial & Yes \\
\hline Haudum et al. ${ }^{(46)}$ & No & No & Yes & Control & Partial & No \\
\hline
\end{tabular}

of available evidence from observational and interventional studies concluded that soy and its components cannot be classified as an endocrine disruptor. These conclusions cover several physiological aspects, including those concerning women's fertility, consistently with the conclusions of this review that highlighted nine additional articles compared with Messina's selection about the topic.

\section{Discussion and conclusion}

Soy isoflavones have repeatedly shown a mild estrogenic effect but at high concentrations they may have enough power to act on hypothalamus and pituitary gland, reducing the ovarian synthesis of estrogens. Furthermore, considering soy as a mere source of isoflavones is extremely reductive. Soy can contain numerous other phytochemicals such as saponins,

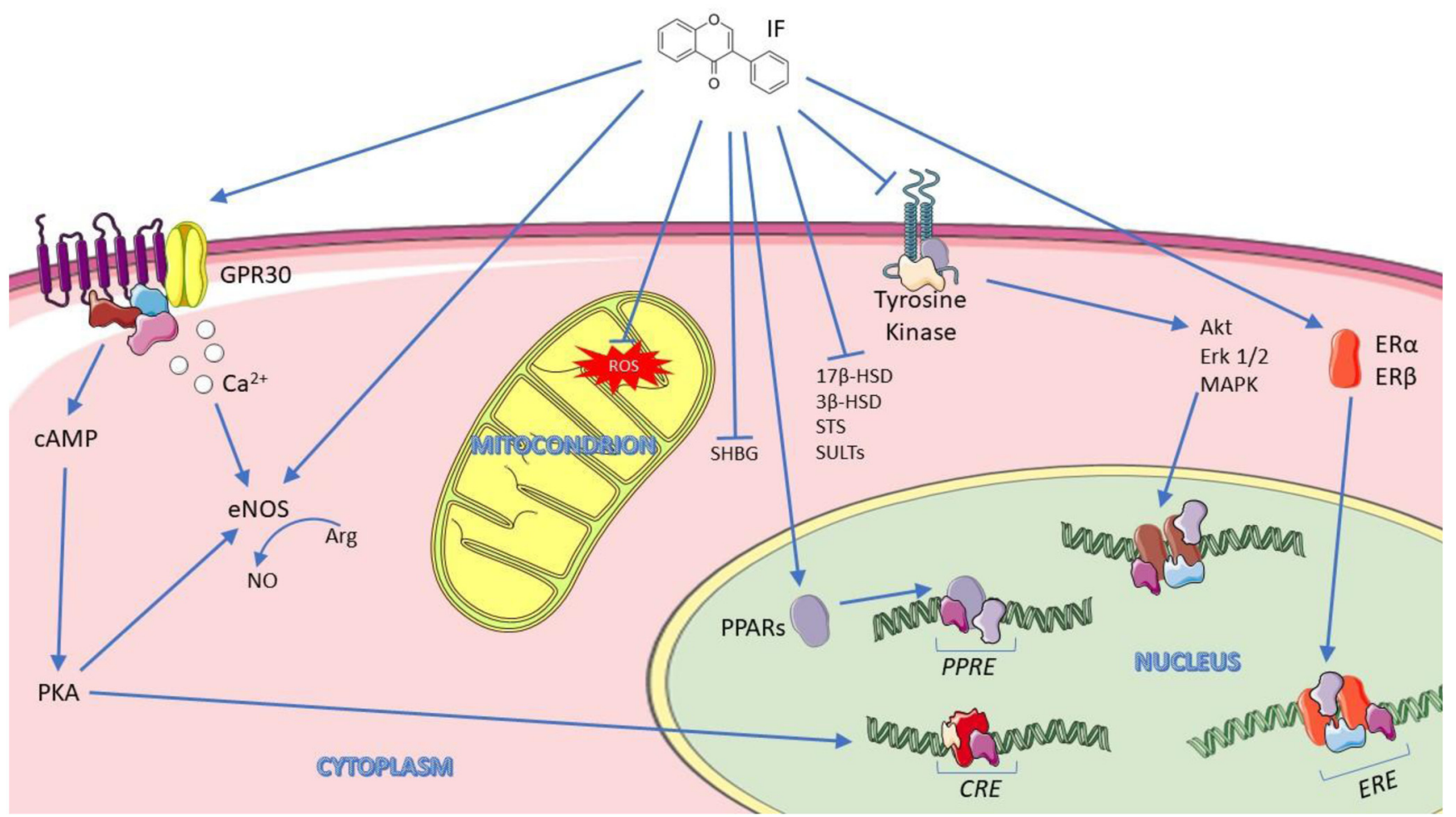

Fig. 2. Main cellular mechanism for isoflavones. Adapted from SMART: Servier Medical Art ${ }^{(89)}$. 
phytosterols, phytic acid, non-isoflavone flavonoids, peptides, protease inhibitors and other bioactive substances.

In a logical perspective, the effect of soy cannot be attributed to the effect of its isoflavones alone. Furthermore, even at high concentrations, they did not show a clear influence on fertility. This could be due to the scarcity of studies on the topic and the presence of few clinical trials, adequately designed to evaluate soy effect on fertility with consistent timing, with an adequate number of participants, blinding, and randomisation for physiological conditions and the presence of equol-producer individuals.

In particular, among selected studies, only the intervention study by Haudum and colleagues explored the stratification of participants for equol-competence ${ }^{(46)}$. In addition, other studies investigated the urinary concentrations of isoflavones and metabolites, including equol ${ }^{(39,45)}$.

Clinical trials can provide solid causal inferences, but they often have limitations in terms of study duration or intervention design. For example, it should be identified whether the interest is related to pharmacological effect, thus implying the use of high concentrations of soy components, or if the aim is to investigate soy functional effects that can be obtained mimicking eating habits, thus providing soy foods with realistic intake levels. The advantages of observational cohort studies include longer times and wider population samples. Nevertheless, these studies often suffer difficulties in evaluating individual effectiveness as well as in identifying possible confounding factors and population characteristics (ethnicity, health conditions, equol-competence, etc.).

In this context, the evaluation of urine samples cannot be underestimated as a valuable tool for detection of the real bioavailability of isoflavones whose metabolisation requires the intervention of intestinal microbiota. Furthermore, it should be considered that, as already discussed, many studies display several limitations including inadequate sampling of hormone concentrations during all phases of cycle, low number of participants and the lack of a placebo group. These aspects considerably reduce the reliability of results, favouring data misinterpretation. Similarly, the stratification by ethnicity and equol-producers may suggest the nature of interactions between soy and fertility. Even if serum AMH concentrations appear as a useful tool for predicting female fertility, only one study from our selection used them ${ }^{(46)}$. Moreover, difficulties related to data collection about nutritional intakes were available, and individual reporting errors must be taken into account. In particular, information about the adequate choice of updated nutritional tables as well as specific nutritional choices, such as increased soy consumption due to pre-existing socio-cultural and physiological aspects should be collected.

To our knowledge, this is the first comprehensive review on soy effect on women's fertility. While soy appears to have a negligible effect on hormonal network, menstrual cycle length and fertility outcomes of healthy women, some clues emerged from literature on its possible beneficial effect in the case of endocrine diseases such as PCOS. Furthermore, the possible ameliorative influence of soy or its components in the case of assisted reproduction techniques outcomes and pregnancy seeking appears promising and worthy of interest.

\section{Acknowledegments}

The authors wish to thank Sandra De Dominici for language revision assistance.

The article processing charge was funded by the Baden-Wuerttemberg Ministry of Science, Research and Art and the University of Freiburg in the funding programme Open Access Publishing.

The authors' responsibilities were as follows: G. R. study conception and drafting the manuscript. A. F. contributed to drafting and revising the manuscript. M. L. contributed to drafting and revising the manuscript. M. A. S. contributed to drafting and revising the manuscript.

The authors declare that they have no conflicts of interest.

\section{References}

1. Rizzo G \& Baroni L (2018) Soy, soy foods and their role in vegetarian diets. Nutrients 10, E43.

2. Ding M, Pan A, Manson JE, et al. (2016) Consumption of soy foods and isoflavones and risk of type 2 diabetes: a pooled analysis of three US cohorts. Eur J Clin Nutr 70, 1381-1387.

3. Jenkins DJA, Blanco Mejia S, Chiavaroli L, et al. (2019) Cumulative meta-analysis of the soy effect over time. J Am Heart Assoc 8, e012458.

4. Nachvak SM, Moradi S, Anjom-Shoae J, et al. (2019) Soy, soy isoflavones, and protein intake in relation to mortality from all causes, cancers, and cardiovascular diseases: a systematic review and dose-response meta-analysis of prospective cohort studies. J Acad Nutr Diet 119, 1483-1500.e17.

5. Schmidt M, Arjomand-Wölkart K, Birkhäuser MH, et al. (2016) Consensus: soy isoflavones as a first-line approach to the treatment of menopausal vasomotor complaints. Gynecol Endocrinol 32, 427430.

6. Taku K, Melby MK, Nishi N, et al. (2011) Soy isoflavones for osteoporosis: an evidence-based approach. Maturitas 70, 333-338.

7. Li N, Wu X, Zhuang W, et al. (2020) Soy and isoflavone consumption and multiple health outcomes: umbrella review of systematic reviews and meta-analyses of observational studies and randomized trials in humans. Mol Nutr Food Res 64, e1900751.

8. Mosallanezhad Z, Mahmoodi M, Ranjbar S, et al. (2021) Soy intake is associated with lowering blood pressure in adults: a systematic review and meta-analysis of randomized double-blind placebocontrolled trials. Complement Ther Med 59, 102692.

9. Rizzo G (2020) The antioxidant role of soy and soy foods in human health. Antioxidants (Basel) 9, E635.

10. Kang J, Badger TM, Ronis MJJ, et al. (2010) Non-isoflavone phytochemicals in soy and their health effects. J Agric Food Chem 58, 8119-8133.

11. Aulisa G, Binda C, Padua E, et al. (2020) If we eat soy, do we keep the beneficial effects of the Mediterranean diet? Nutrition \& Food Science 50, 1099-1108.

12. Basu P \& Maier C (2018) Phytoestrogens and breast cancer: in vitro anticancer activities of isoflavones, lignans, coumestans, stilbenes and their analogs and derivatives. Biomed Pharmacother 107, 16481666.

13. Matthews J \& Gustafsson J-A (2003) Estrogen signaling: a subtle balance between ER alpha and ER beta. Mol Interv 3, 281-292.

14. Liu XX, Li SH, Chen JZ, et al. (2012) Effect of soy isoflavones on blood pressure: a meta-analysis of randomized controlled trials. Nutr Metab Cardiovasc Dis 22, 463-470.

15. Zubik L \& Meydani M (2003) Bioavailability of soybean isoflavones from aglycone and glucoside forms in American women. Am J Clin Nutr 77, 1459-1465. 
16. Iino C, Shimoyama T, Iino K, et al. (2019) Daidzein intake is associated with equol producing status through an increase in the intestinal bacteria responsible for equol production. Nutrients 11, E433.

17. Setchell KDR, Brown NM \& Lydeking-Olsen E (2002) The clinical importance of the metabolite equol-a clue to the effectiveness of soy and its isoflavones. J Nutr 132, 3577-3584.

18. Ariyani W, Miyazaki W, Amano I, et al. (2020) Soy isoflavones accelerate glial cell migration via GPER-mediated signal transduction pathway. Front Endocrinol (Lausanne) 11, 554941.

19. Patel RP, Boersma BJ, Crawford JH, et al. (2001) Antioxidant mechanisms of isoflavones in lipid systems: paradoxical effects of peroxyl radical scavenging. Free Radic Biol Med 31, 1570-1581.

20. Bar-El DS \& Reifen R (2010) Soy as an endocrine disruptor: cause for caution? J Pediatr Endocrinol Metab 23, 855-861.

21. Hamilton-Reeves JM, Vazquez G, Duval SJ, et al. (2010) Clinical studies show no effects of soy protein or isoflavones on reproductive hormones in men: results of a meta-analysis. Fertil Steril 94, 9971007.

22. Reed KE, Camargo J, Hamilton-Reeves J, et al. (2021) Neither soy nor isoflavone intake affects male reproductive hormones: an expanded and updated meta-analysis of clinical studies. Reprod Toxicol 100, 60-67.

23. Liberati A, Altman DG, Tetzlaff J, et al. (2009) The PRISMA statement for reporting systematic reviews and meta-analyses of studies that evaluate health care interventions: explanation and elaboration. PLoS Med 6, e1000100.

24. Moher D, Liberati A, Tetzlaff J, et al. (2009) Preferred reporting items for systematic reviews and meta-analyses: the PRISMA statement. PLoS Med 6, e1000097.

25. Petrakis NL, Barnes S, King EB, et al. (1996) Stimulatory influence of soy protein isolate on breast secretion in pre- and postmenopausal women. Cancer Epidemiol Biomarkers Prev 5, 785-794.

26. Lu LJ, Anderson KE, Grady JJ, et al. (1996) Effects of soya consumption for one month on steroid hormones in premenopausal women: implications for breast cancer risk reduction. Cancer Epidemiol Biomarkers Prev 5, 63-70.

27. Nagata C, Kabuto M, Kurisu Y, et al. (1997) Decreased serum estradiol concentration associated with high dietary intake of soy products in premenopausal Japanese women. Nutr Cancer 29, 228-233.

28. Wu AH, Stanczyk FZ, Hendrich S, et al. (2000) Effects of soy foods on ovarian function in premenopausal women. Br J Cancer 82, 18791886.

29. Lu LJ, Anderson KE, Grady JJ, et al. (2000) Decreased ovarian hormones during a soya diet: Implications for breast cancer prevention. Cancer Res 60, 4112-4121.

30. Strom BL, Schinnar R, Ziegler EE, et al. (2001) Exposure to soybased formula in infancy and endocrinological and reproductive outcomes in young adulthood. IAMA 286, 807-814.

31. Unfer V, Casini ML, Costabile L, et al. (2004) High dose of phytoestrogens can reverse the antiestrogenic effects of clomiphene citrate on the endometrium in patients undergoing intrauterine insemination: a randomized trial. J Soc Gynecol Investig 11, 323-328.

32. Unfer V, Casini ML, Gerli S, et al. (2004) Phytoestrogens may improve the pregnancy rate in in vitro fertilization-embryo transfer cycles: a prospective, controlled, randomized trial. Fertil Steril 82, 1509-1513.

33. Kohama T, Kobayashi H \& Inoue M (2005) The effect of soybeans on the anovulatory cycle. J Med Food 8, 550-551.

34. Romualdi D, Costantini B, Campagna G, et al. (2008) Is there a role for soy isoflavones in the therapeutic approach to polycystic ovary syndrome? Results from a pilot study. Fertil Steril 90, 1826-1833.

35. Khani B, Mehrabian F, Khalesi E, et al. (2011) Effect of soy phytoestrogen on metabolic and hormonal disturbance of women with polycystic ovary syndrome. J Res Med Sci 16, 297-302.

36. Jarrell J, Foster WG \& Kinniburgh DW (2012) Phytoestrogens in human pregnancy. Obstet Gynecol Int 2012, 850313.

37. Filiberto AC, Mumford SL, Pollack AZ, et al. (2013) Usual dietary isoflavone intake and reproductive function across the menstrual cycle. Fertil Steril 100, 1727-1734.
38. Jacobsen BK, Jaceldo-Siegl K, Knutsen SF, et al. (2014) Soy isoflavone intake and the likelihood of ever becoming a mother: the adventist health study-2. Int J Womens Health 6, 377-384.

39. Mumford SL, Sundaram R, Schisterman EF, et al. (2014) Higher urinary lignan concentrations in women but not men are positively associated with shorter time to pregnancy. J Nutr 144, 352-358.

40. Vanegas JC, Afeiche MC, Gaskins AJ, et al. (2015) Soy food intake and treatment outcomes of women undergoing assisted reproductive technology. Fertil Steril 103, 749-755.e2.

41. Andrews MA, Schliep KC, Wactawski-Wende J, et al. (2015) Dietary factors and luteal phase deficiency in healthy eumenorrheic women. Hum Reprod 30, 1942-1951.

42. Chavarro JE, Mínguez-Alarcón L, Chiu Y-H, et al. (2016) Soy intake modifies the relation between urinary bisphenol A concentrations and pregnancy outcomes among women undergoing assisted reproduction. J Clin Endocrinol Metab 101, 1082-1090.

43. Jamilian M \& Asemi Z (2016) The effects of soy isoflavones on metabolic status of patients with polycystic ovary syndrome. $J$ Clin Endocrinol Metab 101, 3386-3394.

44. Wesselink AK, Hatch EE, Mikkelsen EM, et al. (2020) Dietary phytoestrogen intakes of adult women are not strongly related to fecundability in 2 preconception cohort studies. J Nutr 150, $1240-1251$.

45. Levine LD, Kim K, Purdue-Smithe A, et al. (2020) Urinary phytoestrogens and relationship to menstrual cycle length and variability among healthy, eumenorrheic women. J Endocr Soc 4, bvz003.

46. Haudum C, Lindheim L, Ascani A, et al. (2020) Impact of shortterm isoflavone intervention in polycystic ovary syndrome (PCOS) patients on microbiota composition and metagenomics. Nutrients 12, E1622.

47. Wood JW (1989) Fecundity and natural fertility in humans. Oxf Rev Reprod Biol 11, 61-109.

48. Dewailly D \& Laven J (2019) AMH as the primary marker for fertility. Eur J Endocrinol 181, D45-D51.

49. Choung MG, Baek IY, Kang ST, et al. (2001) Isolation and determination of anthocyanins in seed coats of black soybean (Glycine $\max (\mathrm{L}$.$) merr.). J Agric Food Chem 49, 5848-5851.$

50. Oyawoye O, Abdel Gadir A, Garner A, et al. (2003) Antioxidants and reactive oxygen species in follicular fluid of women undergoing IVF: relationship to outcome. Hum Reprod 18, 2270-2274.

51. Kent LM, Morton DP, Ward EJ, et al. (2016) The influence of religious affiliation on participant responsiveness to the complete health improvement program (CHIP) lifestyle intervention. J Relig Health 55, 1561-1573.

52. McBride DC, Bailey KGD, Landless PN, et al. (2021) Health beliefs, behavior, spiritual growth, and salvation in a global population of seventh-day adventists. Rev Relig Res 63, 535-557.

53. Messina M (2010) A brief historical overview of the past two decades of soy and isoflavone research. J Nutr 140, 1350S-1354S.

54. Mumford SL, Steiner AZ, Pollack AZ, et al. (2012) The utility of menstrual cycle length as an indicator of cumulative hormonal exposure. J Clin Endocrinol Metab 97, E1871-E1879.

55. Vassena R, Vidal R, Coll O, et al. (2014) Menstrual cycle length in reproductive age women is an indicator of oocyte quality and a candidate marker of ovarian reserve. Eur J Obstet Gynecol Reprod Biol 177, 130-134.

56. Crawford NM, Pritchard DA, Herring AH, et al. (2017) Prospective evaluation of luteal phase length and natural fertility. Fertil Steril 107, 749-755.

57. Wesselink AK, Wise LA, Hatch EE, et al. (2016) Menstrual cycle characteristics and fecundability in a North American preconception cohort. Ann Epidemiol 26, 482-487.e1.

58. Wise LA, Mikkelsen EM, Rothman KJ, et al. (2011) A prospective cohort study of menstrual characteristics and time to pregnancy. Am J Epidemiol 174, 701-709.

59. Hooper L, Ryder JJ, Kurzer MS, et al. (2009) Effects of soy protein and isoflavones on circulating hormone concentrations in pre- and post-menopausal women: a systematic review and meta-analysis. Hum Reprod Update 15, 423-440. 
60. Kurzer MS (2002) Hormonal effects of soy in premenopausal women and men. J Nutr 132, 570S-573S.

61. Södergård R, Bäckström T, Shanbhag V, et al. (1982) Calculation of free and bound fractions of testosterone and estradiol-17 beta to human plasma proteins at body temperature. J Steroid Biochem 16, 801-810.

62. Meier RK (2018) Polycystic ovary syndrome. Nurs Clin North Am 53, 407-420.

63. Liu R, Zhang C, Shi Y, et al. (2017) Dysbiosis of gut microbiota associated with clinical parameters in polycystic ovary syndrome. Front Microbiol 8, 324.

64. Ferk P, Teran N \& Gersak K (2007) The (TAAAA)n microsatellite polymorphism in the SHBG gene influences serum SHBG levels in women with polycystic ovary syndrome. Hum Reprod 22, 10311036.

65. Escobar-Morreale HF, Luque-Ramírez M \& González F (2011) Circulating inflammatory markers in polycystic ovary syndrome: a systematic review and metaanalysis. Fertil Steril 95, 1048-1058.e1-2.

66. Showell MG, Mackenzie-Proctor R, Jordan V, et al. (2020) Antioxidants for female subfertility. Cocbrane Database Syst Rev 8, CD007807.

67. Pfieffer ML (2019) Polycystic ovary syndrome: an update. Nursing 49, 34-40.

68. Gaskins AJ, Nassan FL, Chiu Y-H, et al. (2019) Dietary patterns and outcomes of assisted reproduction. Am J Obstet Gynecol 220, 567.e1-567.e18.

69. Andres A, Moore MB, Linam LE, et al. (2015) Compared with feeding infants breast milk or cow-milk formula, soy formula feeding does not affect subsequent reproductive organ size at 5 years of age. J Nutr 145, 871-875.

70. Adgent MA, Daniels JL, Rogan WJ, et al. (2012) Early-life soy exposure and age at menarche. Paediatr Perinat Epidemiol 26, 163-175.

71. Sinai T, Ben-Avraham S, Guelmann-Mizrahi I, et al. (2019) Consumption of soy-based infant formula is not associated with early onset of puberty. Eur J Nutr 58, 681-687.

72. Rosselli M, Reinhart K, Imthurn B, et al. (2000) Cellular and biochemical mechanisms by which environmental oestrogens influence reproductive function. Hum Reprod Update 6, 332-350.

73. Zand RS, Jenkins DJ \& Diamandis EP (2000) Steroid hormone activity of flavonoids and related compounds. Breast Cancer Res Treat 62, 35-49.

74. Kuiper GG, Lemmen JG, Carlsson B, et al. (1998) Interaction of estrogenic chemicals and phytoestrogens with estrogen receptor beta. Endocrinology 139, 4252-4263.

75. Ropero AB, Alonso-Magdalena P, Ripoll C, et al. (2006) Rapid endocrine disruption: Environmental estrogen actions triggered outside the nucleus. J Steroid Biochem Mol Biol 102, 163-169.

76. Rowlands DJ, Chapple S, Siow RCM, et al. (2011) Equol-stimulated mitochondrial reactive oxygen species activate endothelial nitric oxide synthase and redox signaling in endothelial cells: roles for F-actin and GPR30. Hypertension 57, 833-840.

77. Akiyama T, Ishida J, Nakagawa S, et al. (1987) Genistein, a specific inhibitor of tyrosine-specific protein kinases. J Biol Chem 262, 55925595.

78. Valles SL, Dolz-Gaiton P, Gambini J, et al. (2010) Estradiol or genistein prevent Alzheimer's disease-associated inflammation correlating with an increase PPAR gamma expression in cultured astrocytes. Brain Res 1312, 138-144.

79. Harada K, Sada S, Sakaguchi H, et al. (2018) Bacterial metabolite $\mathrm{S}$-equol modulates glucagon-like peptide-1 secretion from enteroendocrine L cell line GLUTag cells via actin polymerization. Biochem Biophys Res Commun 501, 1009-1015.

80. Rice S \& Whitehead SA (2006) Phytoestrogens and breast cancer promoters or protectors? Endocr Relat Cancer 13, 995-1015.

81. Nynca A, Sadowska A, Orlowska K, et al. (2015) The effects of phytoestrogen genistein on steroidogenesis and estrogen receptor expression in porcine granulosa cells of large follicles. Folia Biol (Krakow) 63, 119-128.

82. Whitehead SA \& Rice S (2006) Endocrine-disrupting chemicals as modulators of sex steroid synthesis. Best Pract Res Clin Endocrinol Metab 20, 45-61.

83. Gunnarsson C, Ahnström M, Kirschner K, et al. (2003) Amplification of HSD17B1 and ERBB2 in primary breast cancer. Oncogene 22, 34-40.

84. Thompson EA \& Siiteri PK (1974) Utilization of oxygen and reduced nicotinamide adenine dinucleotide phosphate by human placental microsomes during aromatization of androstenedione. $J$ Biol Chem 249, 5364-5372.

85. Mousavi Y \& Adlercreutz H (1993) Genistein is an effective stimulator of sex hormone-binding globulin production in hepatocarcinoma human liver cancer cells and suppresses proliferation of these cells in culture. Steroids 58, 301-304.

86. Déchaud H, Ravard C, Claustrat F, et al. (1999) Xenoestrogen interaction with human sex hormone-binding globulin (hSHBG). Steroids 64, 328-334.

87. Wei H, Wei L, Frenkel K, et al. (1993) Inhibition of tumor promoter-induced hydrogen peroxide formation in vitro and in vivo by genistein. Nutr Cancer 20, 1-12.

88. Morton MS, Arisaka O, Miyake N, et al. (2002) Phytoestrogen concentrations in serum from Japanese men and women over forty years of age. J Nutr 132, 3168-3171.

89. SMART [Internet]. Servier Medical Art. [cited 2021 Jul 26]. Available from: https://smart.servier.com/

90. Messina M, Mejia SB, Cassidy A, et al. (2021) Neither soyfoods nor isoflavones warrant classification as endocrine disruptors: a technical review of the observational and clinical data. Crit Rev Food Sci Nutr, 27, 1-57. 